such topios is to a large extent a personal one, and is naturally related to the research interests of the author. The treatment throughout is that of the pure mathematician. Definitions are precise, arguments are rigorous and there is no concern with applications. The opening sentences of the book define a graph in terms of an edgeset and a vertex-set which are not necessarily disjoint, and load one to expoct that diagrams of points and lines may not be used to represent graphs. The treatment is not, however, as severe as this and diagrams do illuminate the text in a substantial way.

The book is well written and, like most books on graph theory, demands no previous mathematical knowledge (even the term "group" is dofined). The book is not altogether easy reading becauso the style is that of a research paper with close suceessions of definitions and precise proofs. There are several places, however, where the intuitive attractiveness of the research problems breaks through the formal rigorous troatment. This is particularly so in the chapter on girth and $m$-cages.

Research workers in graph theory as a part of pure mathematies will find much to interest them in Professor Tutte's individual treatment and will look forward to the two further volumes of the set.

H. O. Foulkes

\section{ELECTRICITY AND FLUIDS}

\section{An Introduction to Magnetohydrodynamics}

By P. H. Roberts. Pp. vii + 264. (London: Longmans, Green and Co., Ltd., 1967.) 608.

THIs is a useful addition to the growing library of magnetohydrodynamic textbooks at the advanced undergraduate and postgraduate level. The bias of the book is mathematical, for its origins lie in a postgraduate course for mathematicians. Fortunately, some of the lecture room atmosphere comes across in the book, both in the preliminary comments, which open each chapter, and the way in which the topics are outlined and the central problems posed.

As the author admits, the scope of material he has discussed is strictly limited, and he has preferred to deal with a few problems in detail, rather than to give a general review of those subjects amenable to magneto-hydrodynamic treatment. Within these limits, however, a wide range is possible. The emphasis is geophysical and astrophysical-plasma physics enters only in theoretical exercises on hydromagnetic equilibrium and stability - and a chapter on compressible flow and magneto-gasdynamics would have been welcome.

The essontials of the subject are given in the first two chapters and the rest of the book is devoted to their application to specific problems. These introductory chapters on the basic equations and electromagnetic induction are particularly good. Although the formulation is mathematical, the text is illuminated by those comments which physical scientists rarely expect from a mathematician. This measures the success of transposing the lecture course into book form.

The electrodynamie effect of the conducting fluid on the magnetic field through which it flows is considered in chapters on induction and dynamo theory. 'I'his latter chapter displays Professor Roberts's geophysical research interests.

The other aspect of the fluid magnetic field relationship, that is, the hydrodynamic effect of the magnotic stresses on the conducting fluid motion, follows in the scction on hydromagnotic equilibrium, where several magnetic field configurations, familiar in plasma containment experiments, are reviewed.

Both sides of the interaction, the effect of the fluid motion on the field and of the fiold on the fluid, are then considered simultaneously in a discussion of Alfvén wave phenomena. The book is completed by chapters on boundary layer theory, experiments on incompressible flow and on stability theory and its applications. Each chapter ends with a comprehensive set of problems.

The prico is reasonable for a hard-back edition, but the book will reach many more students if it appears as a paperback.

JOHN PAIN

\section{PLASMA RADIATION}

\section{Radiation Processes in Plasmas}

By George Bekefi. Edited by Sanborn C. Brown. (Wiley Series in Plasma Physies.) Pp. xiii +377 . (London and New York: John Wiley and Sons, Ltd., 1966.) $125 s$.

JUST over ten years ago the first book on plasma physics as a subject in its own right appeared; in a gradually swelling stream they have been pouring out ever since. Most of these books have been introductory; there have been surprisingly few monographs. One topic which has had scant coverage in any form is plasma radiation (except for spectral-line radiation which has been dealt with very. fully in Griem's Plasma Spectroscopy). Professor Bekefi's book has now filled this gap.

Radiation Processes in Plasmas begins by recalling some results in the transport of radiation and generalizing classical radiation theory to anisotropic, non-thermal media before going on to discuss bremsstrahlung using a binary collision model; quantum modifications to the bremsstrahlung are summarized. The restriction to binary collisions is then relaxed and a more general point of view adopted by establishing the fluctuationdissipation theorem and applying it to the fluctuations of the electromagnetic field. The dispersion relation for longitudinal waves in a thermal plasma and the damping suffered by these-the Landau damping-are compared with recent experimental results and the treatment of fluctuations ends with an extension of the fluctuationdissipation theorem to plasmas not in thermal equilibrium - more often than not the case in practice.

The reader is now equipped to tackle the problem of radiation from plasmas more realistically. The considerations of bremsstrahlung introduced earlier may now be extended to bremsstrahlung from collective Coulomb interactions rather than from binary encounters alone. Some recent theoretical results on bremsstrahlung from plasmas which, though stable, contain a flux of suprathermal electrons are included on the grounds that they provide a possible model (one of many !) for the type II and type III bursts of radiation from the Sun in its active phase. The bremsstrahlung comes, in this case, from the conversion of longitudinal plasma waves to radiation by scattering on the ions in the plasma; this coupling of longitudinal wave energy to the radiation field on a macroscopic scale (for example, through density gradients) is usually more important in laboratory plasmas and is treated in some detail.

The relaxation of the restriction that the plasma be free from magnetic fields adds enormously to the wealth of radiation phenomena. Again it is simpler to ignore collective offects and, to begin with, a single particle model is considered. Cyolotron radiation by non-relativistic electrons, by highly relativistic electrons (with applications to cosmic radio waves) and by "mildly" relativistic electrons (the regime of many fusion experiments) is evaluated before turning to the drastic changes in the emission spectra which appear once collective effects are taken into account.

The development then turns from the emission and absorption of radiation to the scattoring of electromagnetic waves by plasma fluctuations. The spectrum of density fluctuations is dorived for an isotropic, collisionless plasma and used to treat Thomson scattoring and co-operative scattering both from thermal plasmas and those in which, 